Article

\title{
The Theory of Cognitive-Conditional Conservatism in Accounting
}

\author{
Rodrigo de Oliveira Leite ${ }^{1, *}$ and Ricardo Lopes Cardoso ${ }^{2}$ \\ 1 COPPEAD Graduate School of Business, The Federal University of Rio de Janeiro, \\ Rua Pascoal Lemme 355 Office 423, Rio de Janeiro 21941-902, Brazil \\ 2 Brazilian School of Public and Business Administration, Getulio Vargas Foundation, \\ Rua Jornalista Orlando Dantas, 30, sala 206, Rio de Janeiro 22231-010, Brazil; ricardo.cardoso@fgv.br \\ * Correspondence: rodrigo.oliveira@coppead.ufrj.br
}

Received: 25 August 2020; Accepted: 7 September 2020; Published: 10 September 2020

check for updates

\begin{abstract}
Literature from multiple fields in psychology and economics have identified that impulsive individuals are more prone to riskier behavior and are less conservative. Accounting literature has studied conservatism for many years, and demonstrated that there are two roots of conservatism, one unconditional and another conditional to news available at decision-making. However, there is no bridge linking both. Using an analytical model, we show that the conservatism level of an accountant is lower for impulsive individuals because of their reduced focus on future consequences of their decisions, which is coupled with an increased focus on present consequences. Hence, we put forward a theory of "cognitive-conditional conservatism", that is, a third root of conservatism. Additionally, we also prove the asymmetry property of this behavior.
\end{abstract}

Keywords: accounting conservatism; cognitive reflection; cognitive-conditional conservatism

\section{Introduction}

The conservatism effect is rather ubiquitous in the accounting literature [1] and several papers have shown that some cultural or educational backgrounds can affect this conservatism trait [2-5]. Conservatism has been misunderstood as a pessimistic bias in accounting, and a cause of misreporting financial information [6].

Additionally there is evidence that cognitive reflection can impact the career of accountants in financial industries [7] and that analyses of financial analysts depend on such cognitive reflection traits [8].

In the field of economics, References [9-11] have demonstrated that impulsive people are more conservative than their reflective peers. One of the first investigations was in Reference [12], which asked participants the following question: "There are two urns; each one contains ten balls. Urn A contains 7 red and 3 blue balls, while urn B contains 3 red and 7 blue balls. One urn is randomly chosen by flipping a fair coin. 12 balls are now drawn from this urn with replacement. The result is the following: 8 red and 4 blue balls were drawn. What is the probability that the randomly drawn urn is urn A when observing this result?" The correct probability that the randomly drawn urn is urn A is about $97 \%$, which is derived by Bayes' rule, but impulsive individuals overwhelmingly choose $50 \%$ as their choice [10]. Hence, they measured conservatism based on a performance task from outside the accounting domain.

Though few is known about the role played by accountants' cognitive reflection on conservatism decision-making. Moreover, there has been no research showing why impulsive accountants should be more conservative in their probability assessments. 
This short paper fills such gap. Using an analytical model, we show that impulsive accountants are more short-term focused, and hence they are less worried about future consequences of their decisions, which, in turn, makes them less conservative.

Furthermore, we show that the non-linear nature of the recognition of losses makes this effect asymmetrical. This drop-in conservatism is more pronounced in the gains than in the losses.

The paper is structured as follows. The next section presents the analytical model in four parts: we first model an unbiased accountant which has the task of accounting for gains and losses. The next two parts are related to an impulsive accountant also assigned to the task of accounting for gains and losses. Finally, in the conclusion we present our final remarks.

\section{Theoretical Model}

The present analytical model formalizes the idea that cognitive reflection abilities can affect the assignment of numerical probabilistic thresholds to probability expressions in a more or less conservative way. The model considers four utility functions: a reflective accountant in both gain and loss scenarios, and an impulsive accountant in the same scenarios.

\subsection{Model Setup}

The following theoretical model involves a VNM-utility-maximizing accountant that faces the task of assigning numerical probabilistic thresholds to probability expressions deciding about the recognition and/or the disclosure of an event that increases net asset, hereafter referred to as a gain, as described in function (1) (adapted from Reference [13]):

$$
\begin{array}{r}
U_{A}\left(G, U_{C}, U_{O}\right)=u_{A}(G)+\gamma_{C} U_{C}-\mu_{G} U_{o} \\
U_{o}=u_{o}(G)+\gamma_{o} U_{C} .
\end{array}
$$

An accountant's utility $U_{A}$ is determined by his or her intrinsic utility $u_{A}(G)$, which is assumed to be constant for a given gain $G$. The accountant also internalizes the utility from the firm $U_{C}$, which is assumed to be positive for a gain. Nevertheless, the accountant also internalizes the disutility of recognizing or disclosing a gain that may not be realized $\left(U_{O}\right)$ with probability $1 \geq \mu_{G} \geq 0$. This disutility is modeled as a function of an outside utility $u_{0}(G)$, which represents the firm's stakeholders' (such as auditing firms, market regulators, etc.) and stockholders' utilities, which is again assumed to be constant for a given gain $G$, and the firm's utility $U_{C}$. In this model $\gamma_{c}$ is the weight given by the accountant to the firm's welfare for a gain, while $\gamma_{0}$ represents the weight given by the accountant to the firm's welfare when a gain that already recognized is not realized.

Gains and losses are defined in the model, and also in this paper, in economic rather than accounting terms. Thus, gains are accounting events that increase the utility for both the accountant and the firm, while losses decrease the utility for both the accountant and the firm. We also assume that the gain is exogenous to the model and given before the decision on the probabilistic assessment is undertaken.

Before analyzing the model we must state an important assumption as follows:

Assumption 1. The accountant has no malicious intent and will not commit a fraud, that is, "cooking the books". Hence, if $\mu_{G}=1$ the accountant will not recognize a gain.

This assumption is pretty straightforward. In this case, the accountant does not intrinsically want to break the law and fraudulently recognize a gain that has no chance of materializing. This assumption implies that the accountant is conservative (i.e., s/he gives more weight to negative outcomes of the probabilistic recognition than the positive ones). Hence, we have the following lemma: 
Lemma 1. For a sufficiently important gain, if the accountant has no intention of committing fraud, s/he is conservative.

Proof. The accountant's preference for recognizing or disclosing a given gain can be calculated as the first-order derivative w.r.t. $U_{C}$. If the accountant has no intention of committing fraud, s/he will not account for a gain when $\mu_{G}=1$. Suppose that $\partial U_{A} / \partial U_{C} \geq 0$, then $U_{A}>0 \forall \mu_{G} \in[0,1]$ if $u_{A} \geq \mu_{G} u_{0}$. Thus, a necessary condition for the accountant not to disclose a gain is that $\partial U_{A} / \partial U_{C}<0$. Hence, we can state that:

$$
\frac{\partial\left(u_{A}(G)+\gamma_{c} U_{C}-\left[u_{o}(G)+\gamma_{o} U_{C}\right]\right)}{\partial U_{C}}<0,
$$

which is $\gamma_{c}-\gamma_{o}<0$, implying $\gamma_{o}>\gamma_{c}$.

Thus, we show that the accountant is inherently conservative due to the fact that $\mathrm{s} /$ he is not prone to commit fraud by recognizing a gain which does not exist. We now proceed to start analyzing the problem. Remark 1 presents threshold that a reflective account will use to decide whether or not to account for a gain that has a probability $1-\mu_{G}$ of being realized.

Remark 1. For a sufficiently important decision, if $\left(1-\mu_{G}\right)>\left[1-\gamma_{c} / \gamma_{0}\right]$, the accountant will account for a gain.

Proof. We again calculate the accountant's preference for recognizing or disclosing a given gain as the first-order derivative w.r.t. $U_{C}$ :

$$
\frac{\partial U_{A}}{\partial U_{C}}=\gamma_{c}-\mu_{G} \frac{\partial U_{O}}{\partial U_{C}}=\gamma_{c}-\mu_{G} \gamma_{o}
$$

Consequently, the accountant will recognize and/or disclose a gain for an important enough decision if and only if $\gamma_{c}>\mu_{G} \gamma_{o}$, since if $\gamma_{c}<\mu_{G} \gamma_{o}$, then $U_{A}\left(G, U_{C}, U_{O}\right)<0$ for a sufficiently large $U_{C}$. Therefore an accountant will only account for a gain if $\left(1-\mu_{G}\right)>\left[1-\frac{\gamma_{c}}{\gamma_{0}}\right]$. By Lemma 1 , this is always feasible since $\gamma_{0}>\gamma_{c}$.

Thus, Remark 1 shows that, in essence, if the probability of a gain being realized is greater than a "relative cost of being wrong" (i.e., $\left.\left(1-\mu_{G}\right)>\left[1-\gamma_{c} / \gamma_{o}\right]\right)$, an accountant will recognize and/or disclose a gain.

A similar model can describe this decision-making process when an accountant deals with the recognition and/or disclosure of an event that decreases net asset-hereafter referred to as a loss, as described bellow in function (2):

$$
\begin{array}{r}
U_{A}\left(L, U_{C}, U_{O}\right)=u_{A}(L)-\mu_{L}^{2} \gamma_{c} U_{C}+\mu_{L} U_{o} \\
U_{o}=u_{o}(L)+\gamma_{o} U_{C} .
\end{array}
$$

This model is essentially the same as the one presented in (1), but in this case the signs are inverted because the accountant is making a decision of whether or not to recognize and/or disclose a loss. In addition, to account for the asymmetric nature of loss aversion, the term $\gamma_{c}$ is multiplied by $\mu_{L}^{2}$ since the firm will have a larger disutility from recognizing a possible loss than from an almost certain loss (see Reference [14] for a discussion on this asymmetry).

Again, we will employ the same analysis as the previous case in the following remark:

Remark 2. For a sufficiently important decision, if $\left(1-\mu_{L}\right)>\left[1-\left(\gamma_{c} / \gamma_{0}\right)^{-1}\right]$, the accountant will account for a loss. 
Proof. Using the same method as Remark 1 on (2) we have:

$$
\frac{\partial U_{A}}{\partial U_{C}}=\mu_{L} \frac{\partial U_{O}}{\partial U_{C}}-\mu_{L}^{2} \gamma_{c}=\mu_{L}\left(\gamma_{o}-\mu_{L} \gamma_{c}\right) .
$$

If $\gamma_{o}>\mu_{L} \gamma_{c}$ then the accountant will account for such loss. A conservative accountant in this case assumes $\gamma_{0}<\gamma_{c}$ since sign is inverted. As a consequence, the accountant will recognize and/or disclose a loss if $\left(1-\mu_{L}\right)>\left[1-\left(\gamma_{c} / \gamma_{o}\right)^{-1}\right]$, which is the exact opposite of the gain result.

Accordingly, if an accountant assumes a high probability threshold to account for a gain, he or she will assume a low probability threshold to account for a loss. This is what is usually associated with the idea of a "conservative accountant."

\subsection{Introducing Impulsiveness}

In this subsection we model the accountant's cognitive reflection ability effect on the conservatism models described in the previous subsection. We assume that the previous results are the ones that are expected from an accountant that is not impulsive. Thus, we expect those results to hold for reflective accountants.

Before modeling an impulsive accountant, we state the following assumption:

Assumption 2. Impulsive individuals give more importance to immediate returns than future returns.

This assumption has been tested several times in the economic literature, with impulsive people rather preferring immediate returns than future ones [15-17]. For example References [15,17] showed that impulsive people tend to prefer more to receive $\$ 100$ today than $\$ 140$ in the future when compared to their reflective peers.

We tested this assumption again, using a sample that is more related to this paper, that is, accountants. Hence, we surveyed 237 MBA students in Accounting (Mean age $=29.8$ years, $48 \%$ females and 5.1 years of average work experience). We indeed found that impulsive people prefer immediate returns rather than future ones. Participants that were more impulsive preferred $\$ 100$ today, rather than $\$ 140$ in one year $(t=5.47, p<0.001)$. Thus we show empirically that this assumption is rather acceptable and feasible.

Consequently, we model an impulsive accountant's utility function for recognizing and/or disclosing a gain as described by (3).

$$
\begin{array}{r}
U_{A}\left(G, U_{C}, U_{O}\right)=u_{A}(G)+\theta \mu_{G} \gamma_{c} U_{C}-\left(\mu_{G} / \theta\right) U_{o} \\
U_{o}=u_{o}(G)+\gamma_{o} U_{C} .
\end{array}
$$

In the aforementioned model, $\theta$ is a parameter that captures the impulsive nature of this accountant and which can only assume a value greater than one. For that reason, the accountant overestimates the positive present impact of accounting for a gain, but underestimates a future negative impact of this gain not being realized. For simplicity we assume a linear and symmetric effect of $\theta$ (it increases the present impact by a factor of $\theta$ while reducing the future consequences by a factor of $1 / \theta$ ).

By taking the first order condition w.r.t. $U_{C}$ on this model, which accounts for an impulsiveness nature of an accountant, we can put forward the following proposition:

Proposition 1. Impulsive accountants will account for gains if $\left(1-\mu_{G}\right)>\max \left(\epsilon,\left[1-\theta^{2}\left(\gamma_{c} / \gamma_{0}\right)\right]\right)$, which is to say that they are more likely to recognize a gain than their reflective peers.

Proof. Taking the first order condition from (3), we have that:

$$
\frac{\partial U_{A}}{\partial U_{C}}=\theta \gamma_{c}-\frac{\mu_{G}}{\theta} \frac{\partial U_{O}}{\partial U_{C}}=\theta \gamma_{c}-\frac{\mu_{G}}{\theta} \cdot \gamma_{o} .
$$


If $\mu_{G}<\theta^{2}\left(\gamma_{c} / \gamma_{o}\right)$, the accountant will account for the gain. In comparison with the previous condition for the reflective accountant $\mu_{G}<\gamma_{c} / \gamma_{0}$, we can see that it is more likely that an impulsive accountant will recognize and/or disclose a gain. In fact, in an extreme case in which $\theta^{*} \geq \sqrt{\gamma_{0} / \gamma_{c}}$, this accountant would recognize and/or disclose every possible gain. However, due the fact that an accountant will not recognize a gain when $\mu_{G}=0$ (Assumption 1), the accountant will thus recognize a gain if $\left(1-\mu_{G}\right)>\max \left(\epsilon,\left[1-\theta^{2}\left(\gamma_{c} / \gamma_{o}\right)\right]\right)$ for an arbitrarily small $\epsilon>0$.

This result has two main implications. The first one is that the more impulsive an accountant is, the more likely s/he is to account for a gain, since an impulsive accountant will do so if the probability of the gain not being realized, which is expressed by $1-\mu_{G}$, is greater than a threshold which is proportional to the square of the impulsiveness parameter $\theta$.

The second important take-away from the proof of Proposition 1 is that the likelihood of accounting for a gain is not strictly increasing, but rather has a maximum of 1 if $\theta^{*} \geq \sqrt{\gamma_{0} / \gamma_{c}}$. This result will be further explored later in the paper.

Now we analyze the case for an impulsive accountant exercising his or her judgment on a loss scenario, which is described by (4).

$$
\begin{array}{r}
U_{A}\left(L, U_{C}, U_{O}\right)=u_{A}(L)-\theta \mu_{L}^{2} \gamma_{c} U_{C}+\left(\mu_{L} / \theta\right) U_{o} \\
U_{o}=u_{o}(L)+\gamma_{o} U_{C} .
\end{array}
$$

We use the same rational as Proposition 1 to establish the following Proposition:

Proposition 2. Impulsive accountants will account for losses if $\left(1-\mu_{L}\right)>\left[1-1 / \theta^{2}\left(\gamma_{c} / \gamma_{0}\right)^{-1}\right]$, which is to say that they are less likely to recognize a gain than their reflective peers.

Proof. Taking the first order condition from (3), we have that:

$$
\frac{\partial U_{A}}{\partial U_{C}}=\frac{\mu_{L}}{\theta} \frac{\partial U_{O}}{\partial U_{C}}-\theta \mu_{L}^{2} \gamma_{c}=\mu_{L}\left(\gamma_{o} / \theta-\theta \mu_{L} \cdot \gamma_{c}\right) .
$$

The impulsive accountant will recognize and/or disclose a loss if $\mu_{L}<1 / \theta^{2}\left(\gamma_{c} / \gamma_{0}\right)^{-1}$, which is smaller threshold than the one for the reflective accountant: $\mu_{L}<\left(\gamma_{c} / \gamma_{0}\right)^{-1}$. Thus, it is less likely that an impulsive accountant will account for a loss.

The main prediction from this result is-due to the fact that impulsive accountants are less focused on the future consequences of their decisions, consequently less prudent, they may tend to assign lower probabilistic thresholds for recognizing gains and higher probabilistic thresholds for recognizing losses. We will now formalize this prediction in the following proposition:

Proposition 3. The effect of cognitive reflection ability on assigning numerical probabilistic thresholds to probability expressions is asymmetrical, so that the increase in the propensity to disclose a gain is smaller than the increase in the propensity to disclose a loss.

Proof. From the proof of Proposition 1 we have that if $\theta^{*} \geq \sqrt{\gamma_{0} / \gamma_{c}}$, the reflective accountant will recognize almost every possible gain. However, from the fact that $\mu_{L}<1 / \theta^{2}\left(\gamma_{c} / \gamma_{0}\right)^{-1}$ in Proposition 2, there is no $\theta^{*}$ value for which such accountant would never recognize a loss due to the fact that $\theta \geq 1$. Hence, for any $\theta \geq \theta^{*}$, the accountant has a probability arbitrarily close to 1 of disclosing a gain, but has a probability smaller than 1 for disclosing a loss. This proves the proposed asymmetric effect.

Table 1 summarizes the results. 
Table 1. Threshold probabilities.

\begin{tabular}{ccc}
\hline & Gain & Loss \\
\hline Reflective & $1-\gamma_{c} / \gamma_{o}$ & $1-\left(\gamma_{c} / \gamma_{o}\right)^{-1}$ \\
\hline Impulsive & $\max \left(\epsilon,\left[1-\theta^{2}\left(\gamma_{c} / \gamma_{0}\right)\right]\right)$ & {$\left[1-1 / \theta^{2}\left(\gamma_{c} / \gamma_{o}\right)^{-1}\right]$} \\
\hline
\end{tabular}

\section{Discussion of Results}

This paper introduces the theory of "cognitive-conservatism" in accounting on more general theoretic terms. We show that the empirical findings in which impulsive accountants are shown to be less conservative are based on the fact that those impulsive individuals are more focused on short-term consequences of their decision, rather than long-term ones.

The results from the theoretical model showcase important insights for the effect of impulsiveness on the probability assessments of financial reports and also for accounting conservatism.

First, the result from Lemma 1 shows a possible simple explanation for accounting conservatism: under uncertainty in recognizing gains or losses, if the accountant has no intention to commit fraud, $\mathrm{s} /$ he will act in a conservative manner (adopting a more strict threshold for gains when compared for losses).

Moreover, we show that the fact that the disutility on the recognition for losses is non-linear is responsible for the asymmetry on the recognition in gains versus losses. Hence, impulsive accountants are more prone to recognize possible gains than they are about not recognizing possible losses of similar probabilistic chances.

\section{Conclusions}

This research contributes for being the first on showing why such conservative behavior emerges on the accountants' perspective, and also the impact of cognitive reflection ability on this behavior. Future works could seek to understand if other biases may affect the decision-making of a VNM-utility-maximizing accountant.

Author Contributions: Conceptualization, R.d.O.L.; Methodology, R.d.O.L.; Formal Analysis, R.d.O.L.; Writing—original draft, R.d.O.L.; Critical analysis and validation, R.L.C.; Writing—review and editing, R.d.O.L. and R.L.C. All authors have read and agreed to the published version of the manuscript.

Funding: We thank the support from CAPES, Financial Code 001.

Conflicts of Interest: The authors declare no conflict of interest.

\section{References}

1. Basu, S. Discussion of Conditional and Unconditional Conservatism: Concepts and modeling. Rev. Account. Stud. 2005, 10, 311-321. [CrossRef]

2. Doupnik, T.S.; Richter, M. Interpretation of uncertainty expressions: A cross-national study. Account. Organ. Soc. 2003, 28, 15-35. [CrossRef]

3. Doupnik, T.S.; Richter, M. The impact of culture on the interpretation of "in context" verbal probability expressions. J. Int. Account. Res. 2004, 3, 1-20. [CrossRef]

4. Doupnik, T.S.; Riccio, E.L. The influence of conservatism and secrecy on the interpretation of verbal probability expressions in the Anglo and Latin cultural areas. Int. J. Account. 2006, 41, 237-261. [CrossRef]

5. Ho, J.L.; Chang, C.J. Does national culture or professional knowledge affect auditors' probabilistic conjunction judgments? A study of the United States versus Taiwan. Int. J. Account. 1994, 29, 189-205.

6. IASB. The Conceptual Framework for Financial Reporting; IFRS Foundation: London, UK, 2010.

7. Leite, R.O.; Cardoso, R.L.; Jelihovschi, A.P.G.; Civitarese, J. Job market compensation for cognitive reflection ability. Res. Econ. 2020, 74, 87-93. [CrossRef]

8. Cardoso, R.L.; Leite, R.O.; Aquino, A.C.B. The effect of cognitive reflection on the efficacy of impression management. Account. Audit. Account. J. 2018, 31, 1668-1690. [CrossRef] 
9. Oechssler, J.; Roider, A.; Schmitz, P.W. Cognitive abilities and behavioral biases. J. Econ. Behav. Organ. 2009, 72, 147-152. [CrossRef]

10. Hoppe, E.I.; Kusterer, D.J. Behavioral biases and cognitive reflection. Econ. Lett. 2011, 110, 97-100. [CrossRef]

11. Alós-Ferrer, C.; Hügelschäfer, S. Faith in intuition and cognitive reflection. J. Behav. Exp. Econ. 2016, 64, 61-70. [CrossRef]

12. Edwards, W. Conservatism in human information processing. In Formal Representation of Human Judgment; Wiley: New Jersey, NJ, USA, 1968; pp. 17-52.

13. Cronqvist, H.; Yu, F. Shaped by their daughters: Executives, female socialization, and corporate social responsibility. J. Financ. Econ. 2017, 126, 543-562. [CrossRef]

14. Tversky, A.; Kahneman, D. Advances in prospect theory: Cumulative representation of uncertainty. J. Risk Uncertain. 1992, 5, 297-323. [CrossRef]

15. Frederick, S. Cognitive reflection and decision making. J. Econ. Perspect. 2005, 19, 25-42. [CrossRef]

16. Liersch, M.J.; McKenzie, C.R. Duration neglect by numbers-And its elimination by graphs. Organ. Behav. Hum. Decis. Process. 2009, 108, 303-314. [CrossRef]

17. Thomson, K.S.; Oppenheimer, D.M. Investigating an alternate form of the cognitive reflection test. Judgm. Decis. Mak. 2016, 11, 99.

(c) 2020 by the authors. Licensee MDPI, Basel, Switzerland. This article is an open access article distributed under the terms and conditions of the Creative Commons Attribution (CC BY) license (http:/ / creativecommons.org/licenses/by/4.0/). 